\title{
Una metodología desde la teoría de redes para las redes culturales*
}

\author{
A methodology from network theory for cultural networks \\ Uma metodologia da teoria das redes para as redes culturais
}

Fernando Sancho Caparrini ${ }^{\text {a }}$

DOI: https://doi.org/10.11144/Javeriana.uh89.mtrc

Universidad de Sevilla, España

fsancho@us.es

ORCID: https://orcid.org/0000-0003-1660-4098

\section{Resumen:}

Este documento presenta, desde un punto de vista introductorio y orientado a no expertos en teoría de redes, los fundamentos de grafos y redes que permiten proyectar información sobre redes culturales en un contexto formal. Asimismo, describe algunas de las medidas y herramientas más comunes para diseñar mecanismos cuantitativos para el análisis posterior de estas redes. Este artículo no pretende ser un curso ni un tutorial completo sobre teoría de redes, para eso el lector puede acudir a cualquiera de las referencias que se apuntan al final, más bien constituye una introducción orientada a recorrer, en un solo documento, algunos de los detalles que se consideran más importantes y que suelen quedar fuera de las explicaciones iniciales sobre el tema.

Palabras clave: redes culturales, análisis de redes, teoría de grafos, modelado con redes, redes complejas.

\section{Abstract:}

This paper presents, from an introductory point of view and oriented to non-experts in network theory, the fundamentals of graphs and networks that allow projecting information about cultural networks in a formal context. It also describes some of the most common measures and tools to design quantitative mechanisms for the subsequent analysis of these networks. This article does not pretend to be a complete course or tutorial on network theory, for that the reader can refer to any of the references listed at the end, but rather it is an introduction oriented to go through, in a single document, some of the details that are considered more important and that are usually left out of the initial explanations on the subject.

Keywords: Cultural networks, network analysis, graph theory, network modeling, complex networks.

\section{Resumo:}

Este documento apresenta, de um ponto de vista introdutório e dirigido a não especialistas em teoria de redes, os fundamentos de grafos e redes que permitem projetar informações sobre redes culturais em um contexto formal. Ele também descreve algumas das medidas e ferramentas mais comuns para desenhar mecanismos quantitativos para a análise subsequente dessas redes. Este artigo não tem a pretensão de ser um curso ou um tutorial completo de teoria das redes, para isso o leitor pode recorrer a qualquer uma das referências apontadas ao final, mas constitui uma introdução orientada a percorrer, em um único documento, alguns dos detalhes considerados mais importantes e que muitas vezes ficam de fora das explicaçôes iniciais sobre o assunto.

Palavras-chave: redes culturais, análise de redes, teoria dos grafos, modelagem de redes, redes complexas.

\section{Introducción}

Los elementos fundamentales de una red cultural están constituidos, principalmente, por dos tipos de entidades: las personas, agentes humanos que intervienen de una u otra forma en la red, y los objetos culturales, productos generados por esos individuos dentro del contexto cultural considerado. De igual forma, las relaciones hacen parte de este tipo de red; estas se establecen entre entidades de ambos tipos, o entre entidades del mismo tipo, que también son posibles.

Notas de autor

\footnotetext{
${ }^{\mathrm{a}}$ Correo de correspondencia: fsancho@us.es
} 
Estas relaciones juegan un doble papel en esta estructura cultural. Por una parte, son las responsables de las conexiones directas entre las entidades y, por otra, proporcionan conexiones a larga distancia entre todos los elementos individuales de la red —independientemente del tipo al que pertenezcan y su relación directa - . La dificultad de entender la complejidad de las relaciones a larga distancia, derivadas de las conexiones locales, debe situarnos en la búsqueda de mecanismos que, a partir de las nuevas tecnologías, proporcionen herramientas adicionales para potenciar nuestra comprensión de las dinámicas propias de este tipo de redes (Youngman y Hadzikadic, 2014; Berry, 2012; Diestel, 2005; Dorogovtsev y Mendes, 2003).

Como primer paso hacia este objetivo, y para poder abordar análisis cuantitativos y cualitativos sobre las diversas redes culturales que podemos construir, es necesario disponer de un modelo formal (matemático) con el cual sea posible aplicar herramientas bien fundamentadas de una forma robusta, segura y validable. Hasta ahora, la propuesta de formalización de redes culturales con mayores ventajas es la que se basa en la teoría matemática de redes, fundamentada, a su vez, en la ya clásica teoría de grafos (Chartrand, 2012; Brandes y Erlebach, 2005; Buchanan, 2002). La teoría de redes establece definiciones básicas para manipular entidades y relaciones de una forma flexible y natural, y proporciona un conjunto de herramientas validadas para la extracción de conocimiento, a partir del estudio de estas redes.

De acuerdo con lo anterior, este artículo presenta una introducción para no expertos en teoría de redes, en la que se describen los fundamentos de grafos y redes, que permiten proyectar la información de las redes culturales en un contexto formal, y algunas de las medidas y herramientas comunes para diseñar mecanismos cuantitativos para el análisis posterior de estas redes. Este texto no constituye un curso ni tutorial completo sobre teoría de redes, para eso el lector puede acudir a las referencias que se apuntan al final del texto, por ejemplo, Chartrand (2012) o Diestel (2005). El documento tiene el objetivo de recorrer algunos de los detalles que se consideran más importantes y que suelen quedar fuera de las introducciones para no iniciados. También se pueden encontrar introducciones, más o menos generales, aplicadas al campo de las humanidades digitales (Moretti, 2011; Medina, 2014; Grandjean, 2016; Painter et al., 2019).

Además, daremos una visión del protocolo completo para el tratamiento formal de la información proveniente de una red cultural, que pasa por tres etapas fundamentales: generación del esquema de red, proyección de la información cultural y análisis de la red obtenida. Posteriormente, presentaremos las diversas opciones que se encuentran en cada una de estas etapas, y los riesgos a los que debemos hacer frente, derivados de las elecciones tomadas.

Algunos de estos riesgos son puramente técnicos y podrán ser revertidos en iteraciones posteriores, pero existen riesgos fundamentales que pueden afectar profundamente la etapa de análisis y extracción de conocimiento de la red, por lo que es conveniente tener precaución allí donde puedan aparecer. Uno de los más interesantes, y que más desapercibido pasa entre los investigadores de redes culturales, es el de la influencia que tiene el muestreo de la red real en el transcurso de su proyección a una red digitalizada con el fin de ser preservada y analizada. Por esto, daremos algunas notas acerca de los posibles problemas en esta fase del protocolo, a partir del estudio acerca de qué influencia puede tener en etapas posteriores, si no se toman las precauciones necesarias.

\section{Fundamentos de redes}

En muchos fenómenos modelados se logra identificar que los objetos/entidades/individuos son tan importantes como las relaciones/conexiones que se establecen entre ellos. Aunque no es la única $-\mathrm{y}$ podemos encontrar otras aproximaciones a lo largo de la historia de la ciencia que siguen vigentes y con mucho éxito según los objetivos que se persigan-, la teoría de las redes o grafos — que es como se conocen en áreas más cercanas a las matemáticas- constituye la forma más flexible y sencilla para representar esos dos niveles de la realidad: los objetos y sus relaciones. 
En la teoría de redes, el modelo más simple para contener esta información representa estos objetos mediante un conjunto de nodos ( $V$, también llamados vértices), mientras que las relaciones entre ellos se simbolizan al señalar simplemente qué par de nodos son los que conectan por medio de una arista, también llamada enlace o conexión. Así, es habitual encontrar expresiones como "sea $\mathrm{G}=(V, E)$ el grafo...", que significa simplemente que los nodos de nuestra red $G$ forman el conjunto $V$, mientras que las relaciones entre ellos son las explicitadas en el conjunto $E$. De esta forma, lo único imprescindible es ser capaz de identificar cada uno de los nodos en el conjunto $V$ de forma unívoca, por ejemplo, por medio de una etiqueta única, es decir, un identificador para cada nodo, que podría ser un elemento de un alfabeto, un número, o cualquier código que permita reconocerlo (Fig. 1), y así las conexiones se pueden mostrar mediante pares de la forma $(a, b)$, al indicar que el nodo a está relacionado con el nodo $b$ (Estrada, 2011; Lewis, 2009; Hanneman y Riddle, 2005).

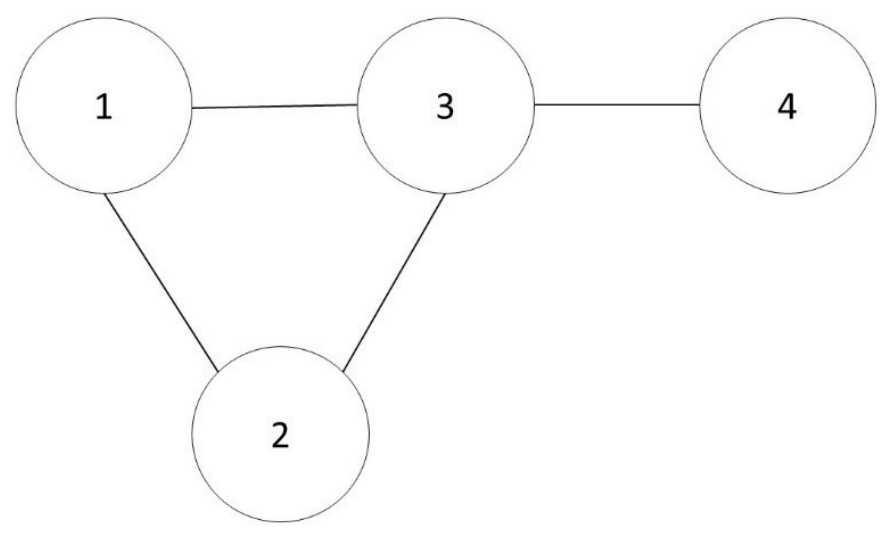

FIGURA 1.

$\mathrm{G}=(\mathrm{V}, \mathrm{E})$, donde $\mathrm{V}=\{1,2,3,4\}$ y $\mathrm{E}=\{(1,2),(1,3),(2,3),(3,4)\}$

Fuente: elaboración propia.

Además, es fácil extraer de aquí una representación gráfica, que es la forma más común de mostrar las redes sencillas de una forma directa y muy intuitiva. Tanto es así que, a menudo, suele confundirse la representación gráfica con la propia red. Puede parecer una estructura demasiado simple para contener información tan importante como la de una red cultural, pero no nos preocupemos por más detalles por ahora, más adelante introduciremos formas de enriquecer la información que se puede reflejar con una red.

Respecto a las relaciones que se consideran en una red, estas pueden ser reales o figuradas, admiten tanta abstracción como el modelador del problema quiera darles. Pueden representar conexiones físicas en el mundo real; por ejemplo, una red de carreteras que conectan entre sí pueblos de una determinada región, enlaces químicos entre átomos de una molécula, etc. También, pueden simbolizar relaciones abstractas que queremos analizar; por ejemplo, podemos conectar dos individuos si hay alguna relación específica entre sus formas de pensar, o reflejar relaciones semánticas entre palabras de un dominio, etc.).

Una de las ventajas más importantes de esta representación es que, una vez figurado el problema real mediante redes, es fácil descubrir que muchas de las preguntas que el fenómeno real nos plantea se traducen en preguntas similares en el modelo matemático. Así, para conocer qué ciudad es la que ofrece mejores condiciones para colocar un centro de distribución de productos, e identificar qué individuo puede haber influido en mayor medida a otros, se requiere un análisis de cada una de las redes asociadas, el número y tipo de conexiones que tienen los nodos que representan esos objetos del mundo real (Abedijaberi, 2018).

Debido a que el mismo modelo matemático puede representar objetos y relaciones tan distintas entre sí, es necesario establecer primero algunas definiciones generales que permitan hablar de los mismos conceptos independientemente del origen del problema y, con estos, buscar soluciones comunes a preguntas planteadas desde dominios y con objetivos completamente distintos. 


\section{Enriqueciendo el modelo básico}

Según la manera en que se considere la arista que une dos nodos, la relación se puede clasificar como dirigida — cuando es importante el orden de cómo la arista conecta los nodos, es decir, el papel que juegan ambos nodos en la relación no es el mismo-, o no dirigida - cuando la arista simplemente conecta los nodos entre sí-. Normalmente, las aristas dirigidas se representan como una flecha (como se observa en la Fig. 2) y las no dirigidas como un segmento.

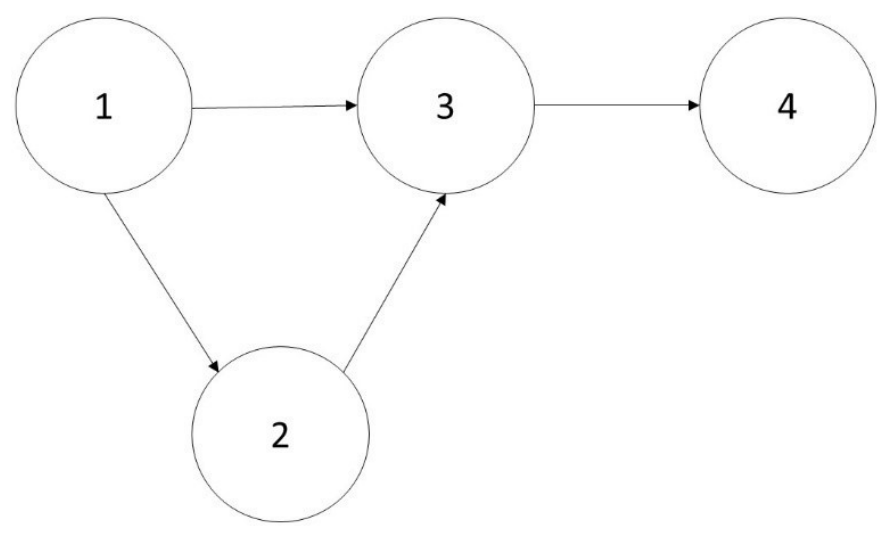

FIGURA 2.

La misma red que la figura 1 , pero con aristas dirigidas

Fuente: elaboración propia.

Desde el punto de vista semántico, usamos aristas dirigidas cuando queremos reflejar que la relación que se da entre los nodos no es simétrica, por ejemplo: A se conecta con $\mathrm{B}$, si el individuo que representa el nodo A es hijo del individuo que representa el nodo B. En este caso, la existencia de una relación de A hacia B, claramente, no implica una relación de $\mathrm{B}$ hacia $\mathrm{A}$. Al contrario, usamos aristas no dirigidas cuando la relación que refleja es simétrica, por ejemplo, en un caso similar al anterior, la relación entre A y B se interpreta como que A y B son hermanos; o bien cuando nos interesa saber que están conectados, pero no el orden que ocupa cada uno en esa relación (Barabási, 2002; Bondy et al., 2008).

Decidir si una relación es de tipo dirigido o no es importante en la etapa de modelado, pues encontraremos posteriormente algoritmos que analizan la red completa y que pueden dar distintos resultados según el tipo de conexiones, pero es una decisión que, si se han tomado las precauciones adecuadas, puede modificarse con facilidad más adelante, incluso considerar una arista como dirigida o no dirigida según el algoritmo que se va a aplicar.

Sin embargo, si queremos usar este modelo para mantener información semánticamente muy rica, como la que reflejan redes culturales provenientes de cualquier contexto, sería muy pobre considerar algo tan sencillo como lo que nos proporciona el modelo básico presentado hasta ahora. En este punto, aparece la potencia del modelo matemático que hay detrás, con el cual es posible añadir variantes de forma muy sencilla a la vez que se mantiene la simplicidad del modelo original.

Hasta ahora solo hemos considerado el caso en que los nodos contienen información acerca de un identificador que lo individualiza frente a los demás nodos del conjunto, pero podemos observar estructuras más ricas dentro de los nodos que permiten mantener información mucho más interesante como, por ejemplo, los datos asociados a una persona, si el nodo representa un individuo del problema real, o los datos de la obra cultural, en este otro caso. Esta información no sustituye al identificador, que es solo una ayuda técnica para manipular el nodo de forma más sencilla, sino que se suma a él y enriquece el contenido del nodo. De esa manera, sería posible, analizar dentro de cada nodo un dato almacenado que evidencie qué tipo de 
entidad del mundo real refleja (persona/obra) y, de esta forma, acercar los nodos a una estructura que almacena información de forma similar a una base de datos.

Algo equivalente se puede considerar en las aristas, en las cuales la información que se almacena no solo puede reflejar qué nodos relaciona, sino datos adicionales acerca de esa relación: tipo, intensidad, antigüedad, etc. De esta forma, las relaciones enriquecidas muestran de forma mucho más natural y potente las relaciones existentes entre los datos de una base de datos relacional clásica, pero con una mayor flexibilidad y con una estructura más potente (Fig. 3).

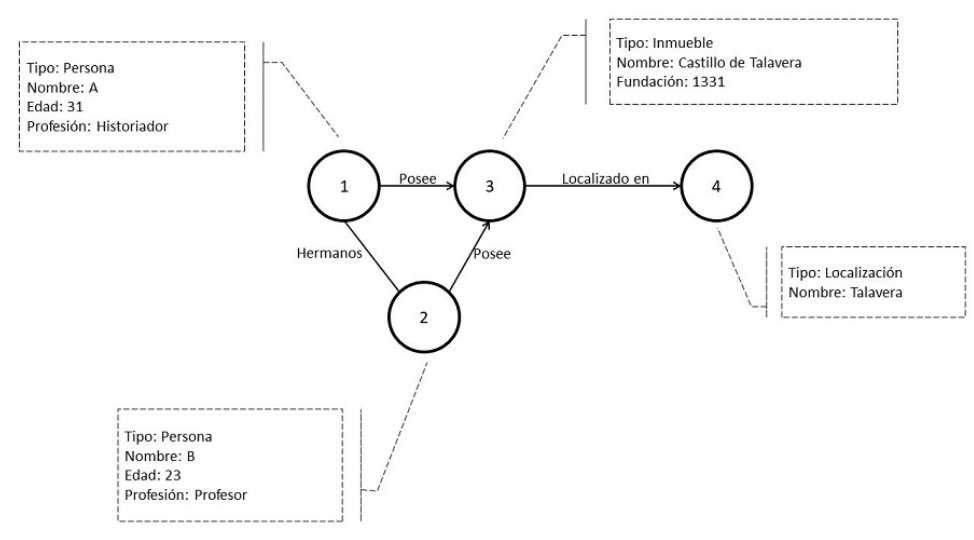

FIGURA 3.

Ejemplo de grafo con propiedades

Fuente: elaboración propia.

A este tipo de redes con información enriquecida, se les conoce en algunos entornos como grafos con propiedades (Rodriguez y Neubauer, 2011), y suponen la base teórica en la que se soportan muchos modelos no estándar de sistemas de información, como las actualmente famosas bases de datos en grafo, que constituyen uno de los ejemplos más exitosos de las conocidas como "bases de datos NoSQL" (De la Rosa et al., 2013).

Aunque en este artículo profundiza en este tema, podríamos haber enriquecido aún más nuestro modelo con un detalle al que no hemos hecho todavía alusión. En los modelos más clásicos, y los más usados e implementados por su mayor sencillez técnica, las aristas se consideran relaciones binarias, es decir, muestran únicamente relaciones entre dos nodos del mundo. Sin embargo, es fácil encontrar relaciones, sobre todo en contextos semánticamente ricos, en las que de forma natural intervienen tres o más entidades; como cuando un individuo realiza una acción tenemos de forma natural una relación binaria entre el individuo y la acción realizada, pero si queremos especificar que un individuo usa un objeto para realizar una acción, la relación precisa ahora de tres entidades para ser reflejada: el individuo, la acción realizada y el objeto usado. Cuando la red admite relaciones no exclusivamente binarias, se dice que estamos ante una hiperred (o hipergrafo), y las aristas pasan a llamarse hiperaristas. En este caso, todo se vuelve un poco más complejo, debido a que ya no es tan sencillo determinar si simplemente son dirigidas o no (la combinatoria de opciones explota), y las herramientas diseñadas para medir y analizar los grafos ya no funcionan igual. Por esa razón, aunque son muy interesantes y sin duda reflejan más fielmente la realidad de muchos problemas, suele ser común restringir el análisis de redes al tipo binario, y convertir las hiperaristas en un conjunto de aristas con un nuevo tipo de nodo artificial, que sirve de nexo común a todos los nodos relacionados originalmente por la hiperarista. A veces es necesario realizar esta operación - que puede ser relativamente complicada y que debe arrastrarse al interpretar todos los resultados que obtengamos en nuestro análisis de la red-, y, a veces, es suficiente introducir información suficiente en la semántica una arista binaria, mientras se sacrifica algo de la riqueza original.

No hay reglas de oro generales, lo fundamental es tomar las decisiones adecuadas con base en el balance de las ventajas y desventajas de cada una de las opciones.

En cualquier texto estándar se pueden encontrar definiciones formales de conceptos tan intuitivos como caminos, ciclos, árboles, grado (número de conexiones de un nodo), etc. Usualmente, estas definiciones no 
tienen en cuenta las estructuras enriquecidas que hemos descrito, es suficiente la estructura básica en la que no hay tipos en nodos ni aristas, pero a partir de ellas es fácil considerar, por ejemplo, caminos al identificar tipos específicos - es decir, no se admite cualquier posible sucesión de nodos y aristas conectadas, sino solo aquellas que siguen patrones específicos-, o grados respecto a tipos - es decir, no se cuentan todas las conexiones de un nodo, sino solo aquellas de determinados tipos-. En esencia, el modelo formal es tan flexible que no debemos sentirnos atados por definiciones estrictas y conceptos prefijados, sino que podemos adaptar formas de interpretar y medir la información de la red a las necesidades de nuestros proyectos e intereses.

\section{Metodología de trabajo}

Si bien el objetivo de este artículo no es profundizar en una metodología de trabajo completa, que ya ha sido presentada con ligeras variantes en otras ocasiones y trabajos (Almagro, 2015), sí puede ser interesante hacer un rápido recorrido por las fases principales, para poder hacer énfasis en algunos elementos que, a veces, pueden pasar desapercibidos.

Recordemos que las tres fases principales que comparten todas las metodologías que se han desarrollado para trabajar con redes en proyectos de humanidades son: generación del esquema de red, proyección de la información y análisis de la red (Fig. 4).

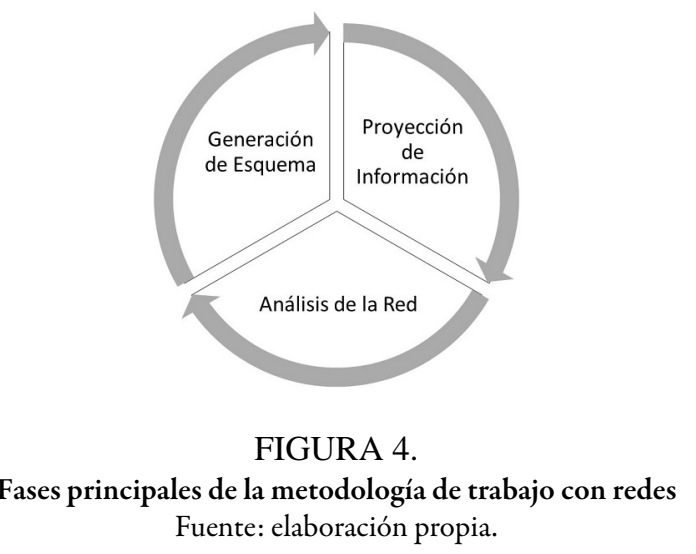

Nos centraremos temporalmente en estas para destacar algunos de sus puntos más interesantes.

\section{Redes con esquema}

Debido a la libertad que ofrecen las redes como soporte conceptual de la información, es posible añadir y relacionar unidades informativas sin seguir un esquema previo, esto es, podemos asociar con absoluta libertad nodos entre sí, agregar propiedades de manera arbitraria e individualizada, e incluir el número de relaciones que queramos entre distintos nodos, cada una con sus propiedades y tipos (Suárez y Sancho, 2013).

Esta libertad, aunque inicialmente atractiva, puede provocar también algunos inconvenientes posteriores, y dificultar la recuperación de la información almacenada y la extracción de conocimiento. Por esto, suele ser habitual que, mediante el uso de la clasificación por tipos de nodos y relaciones semánticas habituales que hay entre ellos y considerando aquellas que sean relevantes para el problema en cuestión, se trabaje sobre un esquema inicial que restrinja de alguna forma qué tipos de nodos y propiedades se pueden crear y cómo se pueden relacionar entre sí (Fig. 5). 


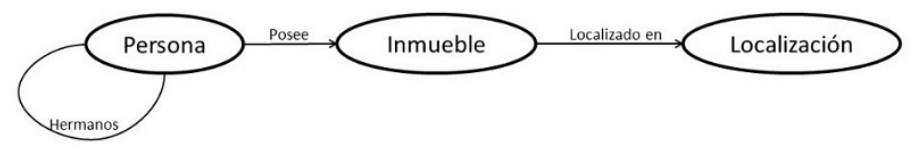

FIGURA 5.

Ejemplo de esquema de una red

Fuente: elaboración propia.

Debe tenerse presente que estas restricciones no son propias del modelo de redes, sino que las incluimos para aumentar el control sobre el modelo.

Esta decisión de diseño no es definitiva, si se trabaja con la herramienta adecuada. Se pueden realizar aproximaciones sucesivas en el esquema definido, a medida que vayamos pasando por las diversas fases del proceso e identifiquemos las carencias encontradas. Si la herramienta no es la adecuada, la adaptación a un nuevo esquema, por pequeña que sea la diferencia, puede suponer una carga de trabajo lo suficientemente grande como para que sea aconsejable detenerse el tiempo suficiente en esta primera etapa, con el fin de estar seguros de que la información almacenada en nuestra red tiene todos los elementos necesarios para responder a las preguntas de investigación. Si, por el contrario, la herramienta facilita la evolución del esquema, entonces, la libertad de ir adaptando este a nuestros objetivos será de un valor incalculable (Suárez et al., 2013).

\section{Proyección de la información}

Esta segunda etapa es equivalente al proceso de población de una base de datos clásica y consiste en, siguiendo el esquema anterior, crear los nodos y aristas que contienen la información concreta de las entidades y relaciones que dan cuerpo a la red real analizada. No nos detenemos en las posibles herramientas que hay disponibles para realizar esta tarea —algunas de acceso directo y otras funcionan por medio de la importación desde otras fuentes de información-, pero sí queremos hacer hincapié en una parte muy importante, y a veces desatendida, que acompaña obligatoriamente esta etapa: el proceso de muestreo.

Necesariamente, las redes digitales son versiones simplificadas de la realidad. Un primer paso de esta simplificación se produce en el momento de crear un esquema reducido de la cantidad de información almacenada de los objetos reales que representan nuestros nodos y relaciones. Esta reducción no responde únicamente a una imposibilidad real de almacenar el mundo completo tal y como es, sino que también tiene como objetivo destacar ciertos patrones en la información que, de otra forma, pasarían inadvertidos, por lo cual resulta extremadamente complicado extraer interpretaciones y conclusiones.

Además de la simplificación estructural que supone reducir el esquema, nos enfrentamos a una simplificación respecto a la sección del mundo real, que proyectamos en nuestra red digital. Necesariamente, a la hora de introducir datos en nuestro sistema gestor de información que almacena la red, realizamos una selección de qué nodos deben ser creados y cuáles no, qué relaciones deben considerarse y cuáles no. Estas decisiones, a veces, responden a algo tan simple como nuestra accesibilidad a la información real. En otras ocasiones, se elige según lo que creemos importante para ser almacenado en la red. Decisiones, voluntarias 
o no, que pueden introducir un sesgo en la información almacenada y, consecuentemente, en las posteriores conclusiones que extraigamos de ella.

Por ejemplo, supongamos que estamos en el proceso de construcción de una red digital cultural con la cual se pretende reflejar la creación artística de un periodo determinado. En el proceso de poblar la red será normal que acudamos a la información disponible en otros medios, como son libros de arte reconocidos, otras bases de datos con buenas valoraciones, catálogos de exposiciones curados por expertos prominentes, etc. Este proceso no solo implica una selección previa de qué se incluye y qué no, sino que es muy probable que en muchas de esas fuentes se sigan criterios similares para destacar unas unidades de la red cultural original frente a otras (Vanrell et al., 2015).

Se sabe que si seguimos un cierto protocolo de selección — por ejemplo, ponderado por el reconocimiento asignado por otras fuentes a los nodos-, la red que construiremos clonará una estructura que justificará la importancia de esos nodos dentro de la propia red, por lo que no podemos estar seguros de si los resultados que estamos obteniendo son objetivos o reflejan el procedimiento de muestreo seguido. Esto no quiere decir que las conclusiones obtenidas sean erróneas, sino que se derivan de una justificación previa al propio análisis y es necesario buscar métodos adicionales para justificarlas (Wasserman y Stanley, 1994).

\section{Análisis de la red}

La etapa de análisis es la que permite hallar las explicaciones del proceso completo. Normalmente, no estamos interesados únicamente en usar nuestra red como un almacén digital del mundo real —aunque ya de por sí sea una tarea suficientemente importante-, sino que hemos hecho la proyección digital para hacer uso de herramientas de análisis para extraer conclusiones interesantes del objeto de estudio (Peña et al., 2009; Suárez y Sancho, 2013; Suárez et al., 2013; Youngman y Hadzikadic, 2014).

Además, para sacar provecho de la estructura de red construida no basta con hacer el mismo tipo de análisis estadístico y consultas que se podrían hacer con un sistema de información tradicional, sino que se debe utilizar la información relacional a larga distancia que la red almacena de forma natural. Por esto, aunque podemos hacer uso de la red para hacer una lectura cercana de alguno de los nodos y sus entornos, aquí el interés radica en aquellas aproximaciones que consideran la red como una unidad completa, que afecta a cada nodo y arista y viceversa.

Es fácil que una red almacene cientos de miles de nodos, millones de aristas y tenga varias decenas de tipos de unos y otras. En estas condiciones, una aproximación visual de la red suele ser bastante insatisfactoria, lo que muestra las limitaciones humanas para entender qué está pasando en la red cuando su tamaño supera las cien unidades. Por lo tanto, es fundamental disponer de una batería de herramientas que permitan diseccionar y probar teorías sobre los datos almacenados.

Es importante destacar que el análisis de una red no es una tarea automatizable, y como en todos los procesos de análisis de datos suficientemente complejos, es necesario realizar diversas aproximaciones y comprobar las hipótesis desde distintos ángulos. Así, un primer conjunto de herramientas necesarias es el de aquellas que permiten transformar la red para seccionarla en trozos menores, más simples y más digeribles: los sistemas de consulta y transformación. De forma similar a los sistemas de consulta existentes en las bases de datos relacionales, ya existen algunos sistemas para extraer información de una red mediante consultas en lenguaje natural, o próximo al natural. El resultado suele ser similar al de una base de datos relacional, con la particularidad de que se trata de una nueva base de datos relacional más pequeña. En el caso de las redes, una consulta general va a dar paso a una red más pequeña, más simple, en la que aparecen ciertos tipos de nodos conectados entre sí cuando verifican la consulta realizada (De la Rosa et al., 2013). Esta nueva red se puede interpretar como una proyección simplificada de la red completa y, por lo tanto, suele ser más sencilla de interpretar. Es posible realizar muchas consultas y obtener muchas secciones de nuestra red original, de 
todas ellas, con las medidas que veremos a continuación, podemos extraer información valiosa que nos orienta acerca de propiedades generales de la red global (Abedijaberi, 2018).

Precisamente, el uso de sistemas de consulta pone de manifiesto la importancia de disponer de una red que siga un esquema predefinido, porque es a partir de este esquema de donde se pueden construir sencillamente consultas informativas (Rodriguez y Neubauer, 2011).

Tras estas transformaciones, y con redes que han reducido su complejidad considerablemente, podemos hacer uso de algoritmos de representación que pueden reordenar la información visualmente para destacar las partes más importantes de la red (Fig. 6).

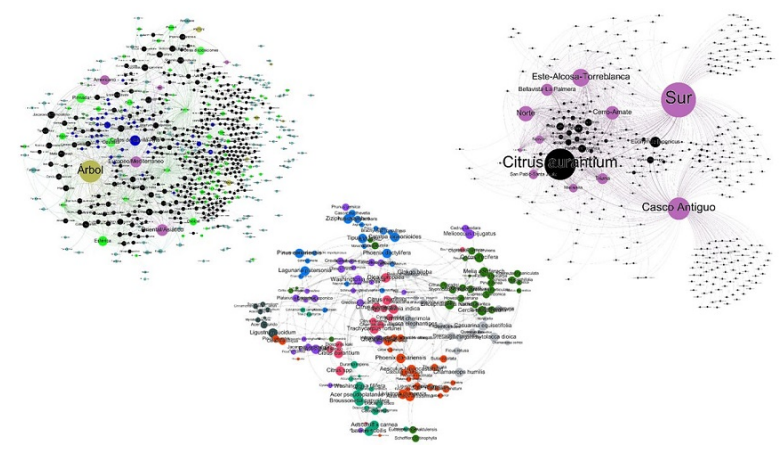

FIGURA 6.

Diversas proyecciones o consultas de una misma red original (Proyecto Jardín Cosmopolita) Fuente: elaboración propia.

En cada una de estas redes derivadas podemos aplicar algoritmos que destaquen de forma objetiva nodos y aristas prominentes para la red. Por ejemplo, los algoritmos de centralidad pueden ayudar a encontrar los elementos más centrales de la red respecto de una medida prefijada; también es posible hallar diversas medidas definidas ya en las librerías de algoritmos sobre redes, cada una muestra características propias (Natarajan, 2018). Por su parte, los algoritmos de comunidades permiten agrupar nodos según su parecido en la red (Javed et al., 2018), es decir, la similitud según cómo se comportan y estructuran dentro de la red que conforman. Un ejemplo paradigmático de centralidad que ha sido aplicado con éxito en entornos humanísticos es el de Page Rank, o algoritmos similares. Este algoritmo fue el que posibilitó la creación e hizo famoso al motor de búsqueda Google, y ayudó en las primeras versiones del buscador a dar un ranking a las páginas web que devolvía el buscador para que el usuario tuviera más oportunidad de encontrar entre los primeros resultados buscados alguno que le pudiera interesar. Se basa en la idea de importancia de un recurso, que brevemente se explica como "un recurso es importante si hay otros recursos importantes que lo referencian" (Langville y Meyer, 2011). Aunque pueda parecer una definición circular, como efectivamente es, esto no quiere decir que esta no sea formalizable y efectivamente calculable. El mismo proceso que siguió Google puede desarrollarse actualmente para procesos como medir la importancia o influencia de un nodo dentro de una red, algo especialmente atractivo cuando estamos trabajando con redes culturales (Fig. 7). 


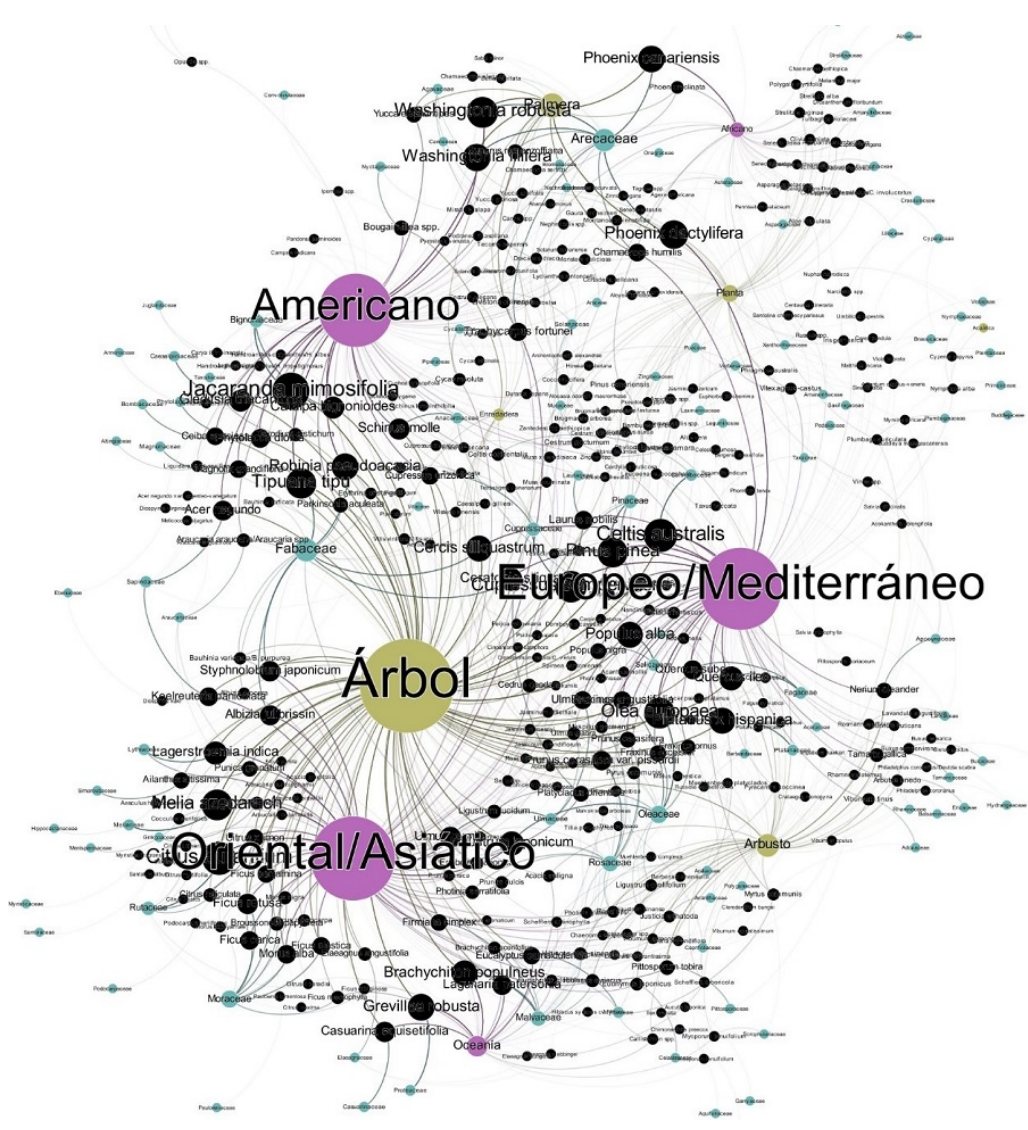

FIGURA 7.

Medición de importancia de cada nodo tras haber realizado una consulta. La importancia de cada nodo se indica por el tamaño de este, para facilitar su interpretación Fuente: elaboración propia.

\section{Conclusiones}

Como cierre de este documento, queremos destacar algunas de las ideas principales que hemos expuesto. Primero, enfatizamos en que el modelo formal matemático de grafos, que da soporte a la aproximación del modelado por teoría de redes, es poderosamente flexible y adaptable. No debemos tener miedo a introducir las variantes que consideremos adecuadas, solo debemos mirar hacia adelante para estar seguros de que el resto de las herramientas que existen van a seguir siendo válidas para nuestro caso. La realidad y la experiencia nos indican que, normalmente, no hace falta inventar un nuevo sistema para cada casuística, y el modelo general de grafos con propiedades es suficientemente simple y potente para contener la gran mayoría de proyectos, sin necesidad de introducir nuevas capacidades.

Segundo, destacamos la importancia de seguir un protocolo para trabajar dentro de un ciclo de vida que podamos controlar. Cuando nuestro proyecto requiere una red es porque la información que va a manejar es suficientemente compleja. En ese sentido, no es deseable introducir más complejidad en tiempo de ejecución por una mala metodología. Ya sean los tres pasos que se han expuesto, o cualquier variante más o menos enriquecida de estos, lo importante es seguir una metodología robusta, validable, y reproducible, como en cualquier proyecto de investigación.

Tercero, es importante recordar que en todo proceso de modelado siempre hay que sacrificar algo del mundo real para tener la capacidad de concluir resultados generales, con cierta validez. Este artículo ha demostrado que este sacrificio se traduce en dos simplificaciones imprescindibles cuando se trabaja con redes: 
restringir el uso de redes a aquellas que siguen esquemas predefinidos — aunque las redes en sí no tienen esta limitación-, y entender que el proceso de población de la red digital implica una decisión de muestreo de los nodos y las aristas de la red real que estamos modelando.

Por último, resaltamos que la terna formada por consultas, medidas y representación proporciona una potente caja de herramientas que, manipulada adecuadamente, permitirá aprovechar la información de las redes analizadas. Actualmente, todavía estamos en la infancia del análisis de redes en general - más aún en el mundo de las humanidades-, pero los resultados que ya arroja esta combinación son prometedores y bien podrían convertirse en una de las piedras angulares de los proyectos cuyo objeto de trabajo es suficientemente complejo. Posiblemente, tengamos que esperar a que se forme una nueva generación de analistas que trabajen con estas herramientas de forma tan natural como actualmente se trabaja con las herramientas clásicas. Cada paso en esa dirección es un paso de gigante hacia un nuevo paradigma de hacer investigación.

\section{Referencias}

Abedijaberi, A. (2018). Mining and analysis of real-world graphs. Missouri University of Science and Technology.

Almagro, P., Dávila, E., Guerrero, G., Medina, I., Regalado, E., Ruíz, Z., Salvador, J. y Sancho, F. (2015). Nuevas líneas metodológicas aplicadas al patrimonio cultural ecuatoriano, Revista del Patrimonio Cultural del Ecuador, 6, 68-75. https://site.inpc.gob.ec/pdfs/Publicaciones/Revista-INPC-\%236-version10-LowRes.pdf

Barabási, A. L. (2002). Linked: The new science of networks. Plume.

Berry, D. M. (2012). Understanding digital humanities. Palgrave MacMillan.

Bondy, J. \& Murty, U. (2008). Graph Theory. Springer-Verlag.

Brandes U. \& Erlebach T. (eds.) (2005). Network analysis: Methodological foundations. Volume 3418 of Lecture Notes on Computer Science. Springer-Verlag.

Buchanan, M. (2002). Nexus: Small worlds and the groundbreaking science of networks. Norton.

Chartrand, G. (2012). Introductory graph theory. Dover books on mathematics. Courier Corporation.

De la Rosa Pérez, J., Suárez, J. L. \& Sancho, F. (2013). SylvaDB: A polyglot and multi-backend graph database and management system. Proceedings of the 2nd International

Conference on Data Technologies and Applications, 285-292. https://doi.org/10.5220/0004475702850292

Diestel, R. (2005). Graph Theory. Springer-Verlag.

Dorogovtsev, S. \& Mendes, J. (2003). Evolution of networks: From biological nets to the internet and www. Oxford University Press.

Estrada, E. (2011). The structure of complex networks. Theory and applications. Oxford University Press.

Grandjean, M. (2016). A social network analysis of Twitter: Mapping the digital humanities community. Cogent Arts \& Humanities, 3(1). https://www.tandfonline.com/doi/full/10.1080/23311983.2016.1171458

Hanneman, R. \& Riddle, M. (2005). Introduction to social network methods. Lecture notes. University of California.

Javed, M.A., Younis, M. S., Latif, S., Qadir, J. \& Baig, A. (2018). Community detection in networks: A multidisciplinary review. Elsevier Science.

Langville, A. N. \& Meyer, C. D. (2011). Google's pagerank and beyond: The science of search engine rankings. Princeton University Press.

Lewis T. G. (2009). Network science: Theory and practice. John Wiley.

Medina, M. A. (2014). Network theory and its applications in arts practice and history of arts. RupkathaJ Interdiscip Stud Humanit, 6(3). https://rupkatha.com/V6/n3/02_Network_theory.pdf

Moretti, F. (2011). Network theory, Plot analysis. A Stanford Lit Lab Pamphlet.

Natarajan, M. (2018). Centrality metrics for complex network analysis. Emerging Research and Opportunities. Advances in Wireless Technologies and Telecommunication. IGI Global. 
Painter, D. T., Daniels, B. C. \& Jost, J. (2019). Network analysis for the digital humanities: principles, problems, extensions. Isis, 110(3), 538-554. https://www.journals.uchicago.edu/doi/full/10.1086/705532

Peña, M., Suárez, J. L. \& Sancho, F. (2009). Topic maps for philological analysis. Linked topic maps. Fifth International Conference on Topic Maps Research and Applications Conference, TMRA, Leipzig, Germany. http://tmra.de/20 09/documents/TMRA2009_Proceedings.pdf

Rodriguez, M. \& Neubauer, P. (2011). A path algebra for multi-relational graphs. 2nd International Workshop on Graph Data Management (GDM'11), IEEE, Hannover, Alemania. https://arxiv.org/abs/1011.0390

Suárez, J. L. \& Sancho, F. (2013). Evolving creativity: An analysis of the creative method in elbulli restaurant. International Conference on Culture and Computing, 2013. 10.1109/CultureComputing.2013.25.

Suárez, J. L., Sancho, F., Ortega, E., De la Rosa, J., Caldas, N. \& Brown, D. (2013). Literary and Linguistic Computing, 28(4), 718-735. https://doi.org/10.1093/llc/fqt050

Vanrell Vellosillo, A., Sancho Caparrini, F., Suárez, J. L. y Sánchez Ortiz, A. (2016). Propuestas para el análisis de colecciones de arte a través de metodologías y herramientas computacionales. Anuario TAREA, 2(2), 136-153. https://idus.us.es/handle/11441/107076

Youngman, P. A. \& Hadzikadic, M. (2014). Complexity and the human experience: Modeling complexity in the bumanities and social sciences. CR Press.

Wasserman, S. \& Stanley, K. F. (1994). Social network analysis: Methods and applications. Cambridge University Press.

\section{Notas}

* Artículo derivado de investigación

\section{Licencia Creative Commons CC BY 4.0}

Cómo citar este artículo: Caparrini, FS. (2020). Una metodología desde la teoría de redes para las redes culturales. Universitas Humanistica, 89. https://doi.org/10.11144/Javeriana.uh89.mtrc 